\title{
In search of the optimal operation for rectal prolapse: the saga continues...
}

\author{
W. C. Cirocco ${ }^{1}$
}

Received: 28 January 2019 / Accepted: 29 January 2019 / Published online: 4 February 2019

(c) Springer Nature Switzerland AG 2019

There have been many radical shifts in the surgical management of rectal prolapse over the past 140 years, a metaphorical seesaw between perineal and abdominal operations. St. Mark's Hospital has played a major role in the surgical management of this disorder following the first case report of perineal proctectomy by Auffret (Brest, France) in 1882 and a small case series of Mikulicz (Konigsborg, Prussia) in 1889, leading to the extensive experience of Miles (St. Mark's, London) with perineal proctectomy, an operation that dominated the first half of the twentieth century, so much so that another St. Mark's surgeon, William Gabriel, suggested the operation be known as the "rectosigmoidectomy- Auffret-Mikulicz-Miles procedure" [1].

The abdominal operations for rectal prolapse came along in the mid and late twentieth century, but were associated with increased morbidity and mortality, before a resurgence of the perineal approach in the first decade of the twentyfirst century, including six published studies with recurrence rates all under $10 \%$ (a total of eight studies at or under $15 \%$ rate of recurrence) [2]. However, the emergence of minimally invasive surgery (laparoscopic and robotic) have energized surgeons to use this technology for rectal prolapse bringing abdominal operations back into the mainstream, even for this typically elderly population of women, with the hope of limiting the morbidity and mortality usually associated with the abdominal approach.

The retrospective review of de Bruijn and associates from St. Mark's, "Long-term outcome of laparoscopic rectopexy for full-thickness rectal prolapse" presents their experience with laparoscopic suspension procedures $(90 \%$ posterior suture rectopexy) to correct rectal prolapse in 80 patients from 2006 to 2014, with 'long-term' follow-up defined as

W. C. Cirocco

william.cirocco@yahoo.com

1 Division of Colon and Rectal Surgery, Department of Surgery, The Ohio State University, N711 Doan Hall, 410 West 10th Avenue, Columbus, OH 43210-1228, USA greater than 12 months [3]. The authors should be commended for their persistence in tracking down these patients and their honesty in reporting the long-term recurrence rate of rectal prolapse. It is well established that the longer the duration of postoperative follow-up after surgical intervention for rectal prolapse, the higher the rate of recurrence, regardless of the method of repair. Furthermore, this was the first operation for only 45 of their 80 patients $(56 \%)$ while the other 35 patients (44\%) had prior operations: a total of 51 total previous operations with the vast majority perineal procedures (40 DeLorme, 6 Altemeier procedures) along with five abdominal operations ( 3 open rectopexy and 2 resection/rectopexy). It is also well established that patients who recur after surgical intervention have an increased rate of re-recurrence with the next operation. Therefore, the large number of patients in the study who suffer from recurrent rectal prolapse should be taken into account when considering the high rate of recurrence of prolapse in this series.

Ninety percent of the operations in this study were laparoscopic posterior suture rectopexy performed by "senior surgeons" with "considerable experience in laparoscopic rectal surgery". The $23 \%$ rate of recurrence of rectal prolapse is disappointing, with the median time to recurrence of 12 months. Of the nine patients who developed recurrence of rectal prolapse in the first 12 months following the operation: five of the nine had previous operations and three of these five patients had multiple prior repairs. It is interesting that the authors chose laparoscopic ventral mesh rectopexy as the secondary procedure for four of the nine patients with early recurrence. Three of these four patients $(75 \%)$ had prompt recurrence of rectal prolapse after laparoscopic ventral mesh rectopexy. Therefore, of the six patients who underwent laparoscopic ventral mesh rectopexy, at least three of these patients (50\%) developed recurrence of rectal prolapse. Ventral or anterior suspension procedures have been around since the 1950s. Originally the mesh was attached to the symphysis pubis [4], but more recent strategies describe attachment of the mesh to the 
sacral promontory [5]. Recurrence rates might be expected to decrease over time as the level of experience of the surgeons performing anterior suspension operations increases.

Of course, besides the primary outcome of recurrence of rectal prolapse, anorectal function is another important outcome to monitor. I agree with the authors that "patients with recurrence may have an inherent tissue weakness and chronic pelvic floor laxity which contribute to recurrence" [3]. Furthermore, this "inherent tissue weakness and chronic pelvic floor laxity" is not specifically addressed by any of the abdominal operations previously described for rectal prolapse, including the authors preferred approach (i.e., posterior suture rectopexy) or the ventral mesh rectopexy they performed for recurrent cases, which lead to a high failure rate in this patient population. It is noteworthy that, since the description of the ventral rectopexy technique that the authors have adopted [5], several variations have been described addressing this very issue by anchoring of the mesh to the perineal body [6] and/or to the puborectalis muscle. It is also noteworthy that the perineal approach (e.g., Altemeier) has the advantage of providing access for levatorplasty, originally described by Cohn [7] and adopted by Altemeier, as a means of decreasing the size of the gaping levator hiatus with its weakened, stretched musculature from chronic prolapse of the rectum. This maneuver may not only improve pelvic floor function, but is also thought to decrease rates of recurrent rectal prolapse. Would the best surgical approach perhaps be an abdominal suspension operation combined with a perineal procedure to correct the typical patulous anus and levator floor deterioration, so often found in these elderly women with chronic rectal prolapse?

An old adage states, "There is nothing new under the sun". Indeed, Dunphy [9] reported success in a series of patients who underwent such a combined and staged procedure beginning with a perineal approach (proctectomy) followed by an abdominal suspension operation a few days later during the same hospitalization. The nest to last patient in this series, however, did not have the second (abdominal suspension) stage of the operation "because of the advanced age of the patient, 72 years, the abdominal operation has been postponed indefinitely" [9]. The patient was discharged and had an excellent recovery without recurrence. Dunphy [9] published this experience in 1948 and the success of the next to last patient in the series, who had only the first stage of the operation (perineal protectomy), was the impetus for Altemeier's "One stage perineal repair of rectal prolapse" [8], a strange title if one is not aware of the 'backstory' of Dunphy's [9] published two-stage repair, a study that would lapse into obscurity.

Perhaps it is time to reconsider a combined abdominal and perineal approach? It is worth a second look, given the rise and fall of so many other suboptimal surgical approaches over the past century. One thing is certain, despite the 96 year-old in the deBruijn and associates study who underwent a successful abdominal suspension procedure without incident, not all elderly women are candidates for an abdominal operation, even if performed with a robot or laparoscope. I would suggest keeping a perineal approach, such as the DeLorme or Altemeier procedure, in the surgeon's armamentarium for those infirm patients unfit for a lengthy abdominal operation, minimally invasive or otherwise.

Funding None.

\section{Compliance with ethical standards}

Conflict of interest The authors declare that they have no conflict of interest.

Ethical approval This article does not contain any studies with human participants performed by the author.

Informed consent Informed consent is not applicable for this editorial.

\section{References}

1. Gabriel WB (1958) The treatment of complete prolapse of the rectum by rectosigmoidectomy (Auffret-Mikulicz-Miles procedure). Dis Colon Rectum 1:241-250

2. Cirocco WC (2010) The Altemeier procedure for rectal prolapse: an operation for all ages. Dis Colon Rectum 58:1618-1623

3. de Bruijn H, Maeda Y, Tan K-N, Jenkins JT, Kennedy RH (2019) Long-term outcome of laparoscopic rectopexy for full-thickness rectal prolapse. Tech Coloproctol. https://doi.org/10.1007/s1015 $1-018-1913-z$

4. Nigro ND (1970) A sling operation for rectal prolapse. Proc R Soc Med 63:106-107

5. D'Hoore A, Penninckx F (2006) Laparoscopic ventral recto(colpo) pexy for rectal prolapse: surgical technique and outcome for 109 patients. Surg Endosc 20:1919-1923

6. Tsunoda A, Takahashi T, Hayashi K, Yagi Y, Kusanagi H (2018) Laparoscopic ventral rectopexy in patients with fecal incontinence associated with rectoanal intussusception: prospective evaluation of clinical, physiological and morphological changes. Tech Coloproctol 22:425-431

7. Cohn I (1942) Prolapse of the rectum: a suggested operative procedure for cure. Am J Surg 42:444-449

8. Altemeier WA, Culbertson WR, Alexander JW (1964) One-stage perineal repair of rectal prolapse. Arch Surg 89:6-16

9. Dunphy JE (1948) Combined perineal and abdominal operation for repair of rectal prolapse. Surg Gynecol Obstet 86:493-498

Publisher's Note Springer Nature remains neutral with regard to jurisdictional claims in published maps and institutional affiliations. 